\title{
Cambios en los índices de desarrollo del aprendizaje en niños pequeños con desórdenes del espectro autista
}

Michael Alessandri

Universidad de Miami

\author{
Cheryl Bomba, Anne Holmes \\ Diane Van Driesen \& David L. Holmes \\ Eden Family of Services \\ Miami, Estados Unidos
}

Se midieron los índices de desarrollo del aprendizaje en 20 niños pequeños con desórdenes del espectro autista, con una batería de pruebas objetivas de aprendizaje, lenguaje y adaptación, en un programa de intervención temprana. Estos niños recibieron entre tres y diez horas este programa altamente estructurado, con una estrategia básica de capacitación discreta por ensayo. Los datos obtenidos apoyan la hipótesis de que la intervención temprana con base conductual tiene un impacto tanto clínico como estadísticamente significativo, en los índices de desarrollo de estos pequeños niños con autismo.

autismo / programas / terapia conductual

\section{Changes in developmental rates of learning in young children with autistic spectrum disorders}

The developmental rates of learning of 20 young children with autistic spectrum disorders were measured with a battery of learning, language, and adaptive behavior measures upon entry and exit from an early intervention program. These children received between 3 and 10 hours this highly structured program utilizing discrete trial teaching strategy. These final data support the hypothesis that behaviorally based early intervention can have an impact on developmental rates of learning of these young children with autism, that is both statistically and clinically significant.

Traducción del inglés por José Cuny y Ricardo Braun

Dirección del autor: malessandri@umiami.ir.miami.edu 
Desde la publicación de los resultados del estudio pionero de Ivar Lobas en 1987, se ha investigado ampliamente la posibilidad de que un diagnóstico conductual temprano que lleve a una intervención temprana, produzca una mejora substancial en el funcionamiento de un niño con autismo. Investigaciones posteriores han producido resultados semejantes en niños autistas preescolares que estaban matriculados en programas educativos conductistas (por ejemplo, Anderson, Avery, DiPietro, Edward \& Christian, 1987; Handleman, Harris, Celiberti, Lilleleht \& Tomchek, 1991; Harris, Handleman, Gordon, Kristoff \& Fuentes, 1991; Harris, Handleman, Kristoff, Bass \& Gordon, 1990). Estos estudios demuestran aumentos en lo académico, lenguaje, cuidado personal y desarrollo social, así como incrementos significativos en el CI. De hecho, existe una considerable evidencia que demuestra que "ningún otro tratamiento para el autismo ofrece una evidencia comparativa de efectividad" (Green, 1996, 290).

Como una extensión lógica de esta investigación, los servicios con programas clínicos para niños muy pequeños (menores de tres años) con desórdenes del espectro autista han empezado a utilizar estrategias terapéuticas con base conductual. Debido a que la investigación revela una tendencia hacia el diagnóstico temprano del autismo ${ }^{1}$, estos pro-

1 El Instituto de Investigación de Autismo de San Diego, California, muestra un marcado incre- gramas se enfrentan cada vez más con el reto de ayudar a grupos de niños muy pequeños como para ser considerados en programas preescolares, aun cuando exhiban características conductuales de autismo y retrasos cuantificables del desarrollo. Este estudio exploró el desarrollo y los logros conductuales alcanzados por niños pequeños que asistían al Programa Wawa House Infant and Toddler, una división del Eden Family of Services en Princeton, New Jersey. Este estudio busca contribuir con la literatura especializada, presentando los resultados preliminares sobre niños que empiezan a recibir terapia con base conductual antes de los tres años. El propósito global de este trabajo es doble: (1) presentar una descripción de los servicios de un Wawa House; y (2) presentar los resultados iniciales de aquellos niños matriculados en el programa.

\section{DESCRIPCIÓN DEL PROGRAMA}

\section{The Eden Family Services}

El Eden Family Services es una organización sin fines de lucro en Princeton, New Jersey, que brinda servicios de por vida a niños y adultos con autismo y a

\footnotetext{
mento en el número de niños que reciben diagnóstico de autismo antes de los tres años. Entre 1990 y 1993 , el $8 \%$ de 6.785 niños fueron reportados por sus padres de haber recibido un diagnóstico de autismo antes de los tres años. Y entre 1994 y 1995 , fue el $17 \%$ de 3.916 niños los que recibieron un diagnóstico antes de los tres años (Rimland, 1995).
} 
sus familias. Basados en la concepción de que la mayoría de niños y adultos con autismo necesitarán servicios altamente especializados de por vida, Eden ha desarrollado un conjunto de servicios con base comunitaria y conductualmente orientado, que incluye un programa educativo, opciones de vivienda (casas grupales y departamentos supervisados) y con posibilidades laborales (empleo seguro, con apoyo y competitivo). Sin embargo, a comienzos de los noventa fue identificado un grupo de niños que no recibían atención. Como se describe arriba, un número cada vez mayor de niños menores de tres años era referido a Eden, los que, o bien cumplían con el criterio diagnóstico de autismo, o bien, al parecer, estaban en riesgo de sufrir un trastorno insidioso del desarrollo. Estos niños, que eran muy pequeños para cumplir con los requisitos del programa preescolar de Eden, tenían pocas opciones. Para completar la continuidad de los servicios de por vida, Eden estableció el Programa ${ }^{2}$ Wawa House Infant and Toddler.

\section{Programa Wawa House Infant and Toddler}

El Programa Wawa House Infant and Toddler es un programa de intervención temprana para niños menores de

2 La Familia de Servicios de Eden recibió el mayor aporte financiero para el Wawa House, de Wawa Inc., una empresa que opera en más de 500 supermercados de comida en cuatro estados del este central de Estados Unidos. tres años con diagnóstico de autismo $\mathrm{u}$ otro tipo de desorden insidioso del desarrollo. También pueden participar los niños que experimentan retrasos significativos o disturbios en áreas de la comunicación, socialización, y/o que muestran conductas e intereses típicos. A través de este programa los niños reciben terapia, la que se enfoca en construir la comunicación y las habilidades conductuales de socialización/juego, cognitivas y motoras, con un uso sistemático de los principios del análisis conductual aplicado.

Los niños son vistos en la clínica entre tres y diez horas a la semana ${ }^{3}$. La terapia que se da en forma individual la realiza un equipo multidisciplinario de profesionales, que incluye a educadores para personas excepcionales, patólogos del habla y del lenguaje y terapistas ocupacionales. Desde su ingreso al programa los niños son evaluados en el desarrollo de sus habilidades propias de su edad, en las principales áreas del desarrollo. En consulta con los padres del niño, se desarrolla un Plan de Servicio Familiar Individualizado (IFSP) que apunta a las deficiencias en las habilidades de comunicación, social y cognitiva y desarrollo motor grueso y fino. Las destrezas del cuidado perso-

3 Los niños que cumplen los requisitos para el programa de intervención están financiados a través del Departamento de Salud del Estado de Nueva Jersey hasta por dos horas a la semana. Los gastos adicionales deben ser asumidos por los padres o encargados de los niños. 
nal son enseñadas primariamente por los padres de los niños a través del programa de consulta domiciliaria.

El núcleo del currículum para infantes y niños está compuesto por los siguientes programas diseñados para construir los cimientos del desarrollo de habilidades apropiadas para el aprendizaje según la edad: Contacto Visual; Sentarse Apropiadamente; Imitación Motora Gruesa; Seguimiento de Instrucciones Simples; Estimulación Verbal; Uso de Gestos; Juego; Voltearse; Igualar; Imitación Verbal; Identificación Receptiva de Objeto/Acción; Desarrollo Motor Fino; y Cuidado Personal (comer, vestirse, asearse, etc.). En la medida en que los niños progresan, los programas que se seleccionan son más complejos (Holmes, 1990, 1991), basándose en las áreas individuales de mayor fortaleza y necesidad del niño, y el programa preescolar (a partir de los tres años) que se considera más idóneo para el niño.

\section{Aproximación terapéutica}

La estrategia de intervención primaria discreta usada en el Wawa House (Maurice, Green, \& Luce, 1996; Lovaas, 1981) es la de la enseñanza discreta por ensayo, una estrategia instruccional fundamentada en los principios del análisis conductual aplicado. Se basa en los principios del análisis conductual aplicado. Durante las etapas iniciales de la terapia, los terapistas se centran en construir una relación con el niño y usar los intereses específicos de éste para escoger los materiales y reforzadores a usarse para enseñar las habilidades arriba mencionadas (Dyer, 1989; Koegel, Dyer \& Bell, 1987; Koegel \& Koegel \& Surratt, 1992). Durante este tiempo, los terapistas también trabajan para eliminar las rabietas que tienen algunos niños cuando son puestos en situaciones demandantes (Lobaas, 1981), a través de la extinción y de procedimientos redireccionales y de refuerzo diferencial. En la medida en que los niños adquieren las habilidades necesarias para el aprendizaje (es decir, habilidad de atención, imitar y seguir directivas simples), el nivel de intervención adulta aumenta, sin dejar el paradigma de que "el niño inicia y que el adulto dirige", para asegurarse de que los niños están eligiendo y son reforzados en las actividades terapéuticas.

Los padres reciben apoyo bajo la forma de: visitas a domicilio diseñadas para desarrollar programas específicos con los miembros de la familia en el ambiente natural del niño; terapia en casa dirigida a ampliar la adquisición, generalización y mantenimiento infantil de habilidades; el entrenamiento activo durante y después de cada visita terapéutica, diseñado para darle mayor autoridad al padre para que tenga una mayor dedicación; seminarios diseñados para entrenar a los padres en la necesaria tecnología conductual que incluye tareas y conocimientos; y grupos de apoyo a los padres. 
Además de la recolección de datos de ensayo tras ensayo sobre los programas de adquisición de habilidades durante las sesiones terapéuticas, los niños e infantes del programa Wawa House son sistemáticamente evaluados, utilizando una serie de medidas normativas estandarizadas. Esta evaluación asegura que los niños estén haciendo progresos constantes y cuantifica los métodos terapéuticos resumidos arriba. Antes de que la terapia comience, se le aplica a cada niño/a una batería de tests para evaluar su desarrollo comparado con el de sus pares, teniendo en cuenta las áreas principales de desarrollo (es decir, comunicación, social/emocional, cognitivo/académico, motor grueso y fino y autoayuda). Para los niños e infantes que ingresan al Programa Wawa House, se les administra una batería inicial de pruebas que da una medida de base del rendimiento y se realizan actualizaciones periódicas y evaluaciones de salida que proporcionan una medida objetiva del progreso del niño.

\section{MÉTODO}

\section{Participantes}

Participaron 20 niños en el estudio. El $80 \% \quad(\mathrm{n}=16)$ fueron hombres; $20 \%$ $(\mathrm{n}=4)$ fueron mujeres. Los siguientes criterios fueron establecidos para participar en el estudio: (1) El niño fue diagnosticado como autista o con otro desorden extendido del desarrollo por un evaluador independiente; (2) el niño estaba experimentando un retraso y/o un desorden en al menos dos de las áreas siguientes: socialización, comunicación y conducta; o (3) el niño estaba en riesgo de sufrir un desorden en el desarrollo (es decir, mostrar signos de disturbios sociales, comunicacionales, y/o conductuales, a pesar de que un diagnóstico definitivo aún no se había realizado). En este estudio, todos los participantes reunieron el primer criterio de elegibilidad, esto es que todos tenían un diagnóstico independiente de autismo (45\%) o PDD-NOS (55\%) hecho por un psiquiatra o neurólogo no afiliado al Eden Family of Services o Wawa House. La media de la edad al entrar al programa fue de 27.8 meses con un rango de 20 a 34 meses. La media de la edad al salir del programa fue de 35.2 meses con un rango de 2 a 15 meses.

\section{Pruebas}

Todos los niños fueron evaluados con un protocolo conteniendo cinco pruebas: el Childhood Autism Rating scale (CARS; Schopler \& Reichler, 1990); el Developmental Profile II (DPII; Alpern, Boll, \& Shearer, 1989); el Early Learning Accomplishment Profile (ELAP; Glover, Preminger, \& Sanford, 1988); el Preschool Language Scale (PLS; Zimmerman, Steiner, \& Pond, 1979); y el Vineland Adaptive Behavior Scales-Interview Edition (VABS; Sparrow, Balla, \& Cicchetti, 1984). Es- 
tas pruebas fueron seleccionadas para: (1) determinar en los niños autistas si el número de síntomas y su severidad disminuía con la terapia; (2) cuantificar las ganancias en habilidades debido a la terapia; y (3) determinar si la terapia incrementaba el desarrollo de los índices del aprendizaje. Una combinación de entrevistas e instrumentos de evaluación directa fue seleccionada para proporcionar un amplio perfil del desarrollo de cada niño. Las medidas de la entrevista proporcionaron una descripción de las habilidades que el niño exhibía, mientras que las medidas de evaluación directa mostraron las habilidades que el niño podía manifestar en una situación demandante y también sirvió para validar la información obtenida a través del reporte de los padres.

El CARS es una escala conductual de 15 ítems diseñada para: (1) identificar a los niños con autismo; (2) distinguirlos de aquellos niños con otras desventajas del desarrollo y (3) distinguir a los niños con un rango de autismo leve a moderado, de aquellos niños con autismo moderado a severo. Los ítems representan áreas conductuales diferentes, como Relacionarse con Personas y Comunicación Verbal. Cada ítem evalúa con puntaje de cuatro toda conducta que es severamente atípica para la edad del niño, y con un puntaje de uno la conducta apropiada para la edad. Los valores se asignan a un código numérico para cada área, basados en el criterio previsto y luego son sumados. Puntajes de 37 o más indican un severo grado de autismo; puntajes entre 30 y 36.5 indican un grado de autismo leve a moderado; y los puntajes debajo de 29.5 indican la ausencia de autismo.

El DPII es un instrumento normativo referencial que mide las habilidades del niño a través de cinco áreas del desarrollo: Física, Autoayuda, Social, Académica, y Comunicación. El entrevistador pide a la persona que responda el test, así el niño realice o no ciertas tareas, como subir o bajar escaleras y poner dos palabras juntas para formar una oración. Cada tarea tiene un valor numérico y los ítems son sumados para obtener un puntaje para cada área, expresado en equivalencia de edad.

El VABS es otro instrumento normativo referencial que mide las habilidades del niño a través de cuatro áreas del desarrollo: Comunicación, Vivencia Cotidiana, Socialización y Motora. La persona que responde al test describe al entrevistador las habilidades del niño en las áreas descritas arriba, el que suma cada ítem para obtener un puntaje para cada área del niño. Así como el DPII, los puntajes se expresan en equivalencias de edad. El VABS es usado además del DPII debido a que es más amplio para observar las habilidades que surgen, así como las que el niño ya domina.

El ELAP es normativo referencial, evaluativo de las habilidades del niño a través de seis áreas del desarrollo: Motora Gruesa y Fina, Cognitiva, Lengua- 
je, Autoayuda, y Social Emocional. El evaluador presenta al niño un test de estímulos (juguetes y otros materiales) y tareas específicas para completar, como construir torres de bloques y copiar una línea. El número de tareas aprobadas es sumado y convertido en equivalentes de edad para cada área.

El PLS es también de evaluación directa y mide las habilidades del lenguaje receptivo y expresivo. El evaluador le presenta al niño un test de estímulos (juguetes y dibujos) y tareas específicas para completar, tales como señalar dibujos y nombrar objetos. El número de habilidades que el niño puede demostrar es sumado y convertido a equivalentes de edad.

\section{Procedimiento}

A los padres de los participantes elegibles se les preguntó si deseaban participar en esta investigación. Una vez que se obtuvo el consentimiento, se inició la evaluación de la línea base. La batería completa de evaluación citada arriba se administró a cada familia después de su ingreso al programa. El ELAP y PLS fueron administrados directamente al niño, mientras que el VABS, DP II, y el CARS fueron administrados en un formato de entrevista a los padres del niño (en todos los casos a la madre del niño) Todas las entrevistas, observaciones y evaluaciones directas al niño fueron conducidas por estudiantes doctorales entrenados en evaluaciones de temprana infancia.
Con la finalidad de evaluar con exactitud las capacidades de cada niño, un terapista familiar o padre estuvo presente con el niño y ayudaron en la administración de los tests, proporcionando con entusiasmo premios verbales por tratar de resolver los ítems. Cuando fue necesario se les dio reforzadores tangibles (ítems de comida y juguetes) y también descansos durante los tests. Sin embargo, a pesar de estas estrategias de evaluación individualizadas, la estandarización fue respetada en todos los tests. Se utilizaron materiales estandarizados y no se le permitía a ningún niño varios intentos en algún ítem, a no ser que estuviera específicamente señalado en el manual del test. Adicionalmente, los niños recibieron crédito en los ítems de evaluación directa sólo si mostraban la habilidad durante la sesión de evaluación, a no ser que estuviera específicamente señalado en el manual del test que el ítem podía ser aprobado a través de la información del padre. Ningún niño fue "no evaluable" durante alguna de las sesiones de evaluación y, salvo pocas excepciones, los niños completaron todos los tests en una sesión de una hora. Las entrevistas a los padres fueron hechas estrictamente de acuerdo con el protocolo del manual del test y con los procedimientos estándar para entrevistas psicológicas.

La batería de evaluación fue nuevamente administrada cuando el niño alcanzó los tres años de edad y/o se graduó del programa. 


\section{RESUltados}

Con las muestras apareadas, se hicieron t-tests de las medias del pre y postest con todas las medidas dependientes. Adicionalmente, con las muestras apareadas, se hicieron t-test de la media pretest y postest de los índices de desarrollo de DP II, ELAP, PLS Y VABS. Los índices de desarrollo del pretest fueron calculados dividiendo el nivel de desarrollo pretest por la edad cronológica. Los índices de desarrollo postest fueron calculados dividiendo el cambio en el nivel de desarrollo pre y postest por el número de meses entre el pretest y postest.

Todo los niños mostraron una disminución en la severidad de su sintomatología autista del pretest al postest medido por el CARS; Estas diferencias fueron estadísticamente significativas (véase tabla $\mathrm{N}^{\circ} 1$ ). Se observó mejoras significativas en el desarrollo en todos los subtests del DP II (véase tabla $\mathrm{N}^{\mathrm{o}}$ 2), del ELAP (véase tabla $\mathrm{N}^{\circ} 3$ ), del PLS (véase tabla $\mathrm{N}^{\circ} 4$ ) y del VABS (véase tabla $\mathrm{N}^{\circ}$ 5) del pretest al postest.

Mientras que en la mayoría de puntajes o valores del desarrollo hubo incremento del pre al postest en PD II, sólo en el subtest de Comunicación se produjo un incremento particularmente significativo (véase tabla $\mathrm{N}^{\circ}$ 6). En este subtest, el valor de desarrollo postest fue tres veces mayor que el valor pretest.

Los índices de desarrollo pre y postest se incrementaron en cada subtest del ELAP (véase tabla $N^{\circ} 7$ ). Se obser- vó incrementos significativos en cuatro de los seis subtests: Cognitivo, Motor Fino, Lenguaje y Social. El mayor incremento se observó en las áreas de Lenguaje y Social, con valores postest aproximadamente cuatro veces mayor que los valores de pretest.

En los puntajes de desarrollo del PLS se observó que eran significativamente mayores en el post que en el pretest para todos los subtests (véase tabla $\mathrm{N}^{\circ} 8$ ).

Se notó incrementos en los valores del desarrollo en dos subtests del VABS (Comunicación y Socialización), pero sólo en uno de ellos (Comunicación) fue estadísticamente significativo (véase tabla $\mathrm{N}^{\circ}$ 9). Se observó una disminución en los subtests de Vida Cotidiana y Dominio Motor, alcanzando datos estadísticos significativos sólo en el subtest Motor.

\section{Discusión}

Estos resultados indican que una intervención temprana, a través de un programa estructurado y orientado conductualmente, puede incrementar los índices de desarrollo del aprendizaje en niños pequeños con desórdenes del espectro autista a través de todas las áreas del desarrollo. Es muy importante elevar los índices de desarrollo de niños pequeños con autismo, debido a que mientras la brecha entre estos niños y sus pares normales es relativamente estrecha durante los primeros años del desarrollo, se amplía rápidamente cuando el desarrollo normal se 
acelera, dando como resultado el fenómeno de "correr detrás del tren en movimiento". Mientras que el incremento en los valores del desarrollo no garantiza un resultado en particular, el incremento es crítico si estos niños van a competir con sus pares normales en términos de adquisición de destrezas. Un hallazgo particularmente alentador es que los más dramáticos aumentos ocurren en las áreas de Comunicación y Socialización, dos áreas asociadas con las severas y crónicas carencias en individuos con autismo.

Como en muchos estudios, los autores encontraron dificultades metodológicas. Los resultados de este estudio se habrían fortalecido con datos obtenidos de grupos de control y/o comparativos. Sin embargo, surgieron problemas éticos y logísticos. Primero, aun cuando los autores habían identificado al grupo de niños que no recibían atención, consideraciones éticas habrían impedido a los autores incluir a estos niños en la matrícula en un grupo de no tratamiento. Segundo, los autores no pudieron localizar un número suficiente de niños cuyos padres habían escogido seguir un enfoque no conductista (por ejemplo, un modelo basado en el juego) como un grupo de tratamiento comparativo. Debido a la preferencia de los padres por la intervención conductista, desde hace más de 25 años, ésta es reconocida en New Jersey como el tratamiento estándar para individuos autistas. Este reconocimiento ha dado como resultado un desarrollo de programas públicos y privados que utilizan la enseñanza de ensayo discreto como la estrategia básica de instrucción (Cosak, 1994; Task Force on Autism, 1985). A pesar de que un gran número de padres fue contactado a través de programas genéricos de intervención temprana, la mayoría de sus hijos estaba recibiendo terapia basada en la conducta e intervención temprana. Debemos destacar que un "problema" semejante (para los investigadores, no para los padres) fue encontrado también por Handleman, Harris, Gordon, Kristoff, y Fuentes (1991), investigadores de otra agencia en New Jersey.

Ningún estudio escrito después de 1987 puede evitar referirse al rol de la intensidad ${ }^{4}$, posiblemente "una de las dimensiones más importantes de la intervención conductual" (Green, 1996, 40). La prescripción inicial de Lovaas (1987), de 40 horas semanales como mínimo, ha sido adoptada por numerosos terapistas, programas y agencias, todos esperando poder replicar su hallazgo de "funcionamiento normal" (McEachin, Lovaas \& Smith, 1993) para casi la mitad de niños tratados. Las tres a diez horas de terapia a la

4 Mientras que la intensidad del tratamiento puede ser definida de maneras diferentes (interacciones terapista/niño, número de contactos por semana, número de horas por semana, etc.) en nuestro caso, la intensidad es definida como el número de horas por semana. 
semana recibidas por los niños en el programa Wawa House, no se aproximan al número de horas recibido por los niños en los programas que siguen el modelo de Lobas. Sin embargo, es importante considerar las restricciones en las que actúan muchos padres. La investigación ha documentado que muchos factores impactan en la habilidad de los padres para generar y mantener el cambio en la conducta de sus hijos, que incluyen recursos económi$\cos$ y fuentes de apoyo emocional (Helm y Kozloff, 1986). Implementar un programa en casa, de 40 horas a la semana para un infante o niño con autismo, requiere de los padres un compromiso de por lo menos dos años, y asumir simultáneamente los roles de partidario, educador, recolector de fondos y padre, además de tener una carga financiera (Huff, 1996). Recientemente, Jacobson, Mulick, y Green (1996) prepararon un análisis de costos y estimaron que el costo anual de un programa intensivo en casa era de aproximadamente 33 mil dólares americanos, con "extrapolación a 50 mil... factible y notable". Esta cantidad de dinero excede el costo de una hipoteca para una casa en muchas partes del país. Como se dijo anteriormente, en New Jersey sólo se asigna dos horas a la semana de servicio de intervención temprana; una mayor cantidad de horas debe ser financiada por los padres mismos. No todos los padres tienen recursos financieros suficientes para pagar más horas o, por muchas razones, no están dispuestos a asumir las responsabilidades que este programa exige. El presente estudio muestra que (por comparación) un programa de baja intensidad puede aumentar significativamente los índices de desarrollo en niños con autismo y reducir en forma también significativa la severidad de su autismo. De hecho, en dos de las áreas más críticas, Comunicación y Habilidades Sociales, los valores de aprendizaje se incrementaron dramáticamente. Estos resultados preliminares indican que los padres que eligen matricular a sus hijos en un programa de baja intensidad pueden esperar un progreso mensurable y significativo. Mientras que los niños en este estudio no obtuvieron un funcionamiento normal antes de haberse graduado del programa Wawa House, hasta donde saben los autores, ningún estudio actual reporta un funcionamiento normal obtenido por grupos de niños menores de tres años. Se necesita hacer un seguimiento longitudinal de 20 niños que se describen aquí, para determinar los efectos a largo plazo de este nivel de intensidad. Adicionalmente, Green (1996) destaca que la interacción entre intensidad del tratamiento (alto) y edad (baja) es desconocida, ya que los estudios anteriores se centran en niños de tres años o mayores. Aún se desconoce si las sesiones centradas en adultos podrían demostrar la misma eficacia que la hallada en una población de infantes y niños. Y, finalmente, 
es importante destacar que todos los niños que se graduaron en el programa Wawa House continuaron asistiendo a programas preescolares especializados, incluyendo los de enfoque conductual con 25 o más horas semanales de instrucción de ensayo discreto. La interacción entre la intensidad del tratamiento en intervención temprana y la intensidad del tratamiento durante el período preescolar es también desconocida. Es posible que un programa de intervención temprana de baja intensidad podría ser más apropiado para niños menores de tres años con el propósito de dar un "arranque súbito" en su aprendizaje (es decir, incrementar los índices de desarrollo del aprendizaje), permitiéndoles beneficiarse al máximo de programas preescolares a tiempo completo con base conductista. Tenemos la esperanza de que la investigación futura revelará los efectos interactivos y dará a los terapistas guías para la intensidad del tratamiento. Hasta ese momento, los resultados de este estudio proporcionan apoyo a la hipótesis de que de tres a diez horas por semana de intervención con base conductual, pueden tener un impacto clínico y estadísticamente significativo en los índices de desarrollo del aprendizaje en niños pequeños con autismo.

Tabla $\mathbf{N}^{\circ} 1$

Medias, desviaciones estándar y puntajes de t-test del cars

\begin{tabular}{lccccc}
\hline Medida & Tiempo 1 & Tiempo 2 & $t$ & df & $\mathrm{p}$ \\
\hline CARS & 29.8 & 27.9 & 2.17 & 19 & .043 \\
& $(5.45)$ & $(4.99)$ & & & \\
\hline
\end{tabular}

Tabla $\mathbf{N}^{\circ} 2$

DP II medias (puntajes mensuales), desviaciones estándar puntajes del t-test

\begin{tabular}{lccccc}
\hline Subescala & Tiempo 1 & Tiempo 2 & $t$ & $\mathrm{df}$ & $\mathrm{p}$ \\
\hline Académico & 19.5 & 26.8 & -4.83 & 19 & .001 \\
& $(6.84)$ & $(8.18)$ & & & \\
Comunicación & 15.6 & 25.5 & -7.65 & 19 & .001 \\
& & $(7.61)$ & $(9.15)$ & & \\
Social & 19.9 & 24.2 & -3.51 & 19 & .002 \\
& $(4.92)$ & $(6.89)$ & & & \\
Autoayuda & 21.7 & 25.4 & -3.53 & 19 & .002 \\
Físico & $(5.63)$ & $(6.87)$ & & & \\
& 23.8 & 28.5 & -3.81 & 19 & .001 \\
& $(5.13)$ & $(6.32)$ & & & \\
\hline
\end{tabular}


Tabla $\mathbf{N}^{\circ} 3$

ELAP medias (puntajes mensuales), desviaciones estándar y puntajes t-test

\begin{tabular}{lccccc}
\hline Subescala & Tiempo 1 & Tiempo 2 & $t$ & $\mathrm{df}$ & $\mathrm{p}$ \\
\hline Cognitivo & 12.7 & 20.5 & -6.88 & 19 & .001 \\
& $(7.28)$ & $(6.68)$ & & & \\
Motor Fino & 16.8 & 23.3 & -5.63 & 19 & .001 \\
& $(5.75)$ & $(6.63)$ & & & \\
Motor Grueso & 21.1 & 26.5 & -6.10 & 19 & .001 \\
& $(3.50)$ & $(4.59)$ & & & \\
Lenguaje & 9.42 & 18.5 & -6.07 & 19 & .001 \\
& $(6.79)$ & $(8.22)$ & & & .001 \\
Autoayuda & 15.5 & 19.6 & -4.89 & 19 & .001 \\
Social & $(3.77)$ & $(4.53)$ & & & \\
& 9.90 & 18.3 & -4.41 & 19 & \\
\hline
\end{tabular}

Tabla $N^{\circ} 4$

PLS medias (puntajes mensuales), desviaciones estándar y puntajes t-test

\begin{tabular}{lccccc}
\hline Subescala & Tiempo 1 & Tiempo 2 & $t$ & $\mathrm{df}$ & $\mathrm{p}$ \\
\hline Auditivo & 14.4 & 22.4 & -4.88 & 19 & .001 \\
\multirow{2}{*}{ Verbal } & $(4.34)$ & $(8.56)$ & & & \\
& 14.6 & 21.5 & -6.21 & 19 & .001 \\
Lenguaje & $(3.93)$ & $(7.68)$ & & & \\
& 14.5 & 21.9 & -6.23 & 19 & .001 \\
\hline
\end{tabular}

Tabla No 5

VABS medias (puntajes mensuales), desviaciones estándar y puntajes t-test

\begin{tabular}{lccccc}
\hline Subescala & Tiempo 1 & Tiempo 2 & $t$ & $\mathrm{df}$ & $\mathrm{p}$ \\
\hline Comunicación & 11.6 & 17.1 & -4.79 & 19 & .001 \\
Vida Diaria & $(5.11)$ & $(7.46)$ & & & \\
& 17.5 & 20.4 & -5.78 & 19 & .001 \\
Socialización & $(2.67)$ & $(3.63)$ & & & \\
\multirow{3}{*}{ Motor } & 13.9 & 17.5 & -4.75 & 19 & .001 \\
& $(4.39)$ & $(4.86)$ & & & \\
& 24.6 & 27.5 & -4.06 & 19 & .001 \\
\hline
\end{tabular}


Tabla $\mathbf{N}^{\circ} 6$

DP II medias valores desarrollo pre y postest, desviaciones estándar y puntajes t-test

\begin{tabular}{|c|c|c|c|c|c|}
\hline Subescala & Valor 1 & Valor 2 & $t$ & df & $\mathrm{p}$ \\
\hline Académico & $\begin{array}{c}.70 \\
(.22)\end{array}$ & $\begin{array}{c}1.24 \\
(1.56)\end{array}$ & -1.48 & 19 & ns \\
\hline Comunicación & $\begin{array}{c}.55 \\
(.25)\end{array}$ & $\begin{array}{c}1.79 \\
(1.75)\end{array}$ & -3.19 & 19 & .005 \\
\hline Social & $\begin{array}{c}.72 \\
(.16)\end{array}$ & $\begin{array}{c}.94 \\
(1.42)\end{array}$ & -.68 & 19 & ns \\
\hline Autoayuda & $\begin{array}{c}.78 \\
(.19)\end{array}$ & $\begin{array}{c}.66 \\
(1.04)\end{array}$ & .50 & 19 & ns \\
\hline Físico & $\begin{array}{c}.86 \\
(.17)\end{array}$ & $\begin{array}{c}.78 \\
(.97)\end{array}$ & .349 & 19 & ns \\
\hline
\end{tabular}

Tabla $\mathbf{N}^{\circ} 7$

ELAP medias valores desarrollo pre y postest, desviación estándar y puntajes t-test

\begin{tabular}{lccccc}
\hline Subpuntaje & Valor 1 & Valor 2 & $t$ & df & $\mathrm{p}$ \\
\hline Cognitivo & .45 & 1.10 & -3.92 & 19 & .001 \\
Motor Fino & $(.23)$ & $(.67)$ & & & \\
& .60 & .94 & -2.11 & 19 & .048 \\
Motor Grueso & $(.19)$ & $(.69)$ & -.862 & 19 & $\mathrm{~ns}$ \\
& .76 & .97 & & & \\
Lenguaje & $(.12)$ & $(1.01)$ & -3.73 & 19 & .001 \\
Autoayuda & .33 & 1.37 & -.761 & 19 & $\mathrm{~ns}$ \\
Social & $(.22)$ & $(1.21)$ & & & \\
& .56 & .79 & -2.37 & 19 & .029 \\
\hline
\end{tabular}

Tabla $N^{\circ} 8$

PLS medias valores desarrollo pre y postest, desviaciones estándar $y$ puntajes t-test

\begin{tabular}{llllll}
\hline Subescala & Valor 1 & Valor 2 & $\mathrm{t}$ & $\mathrm{df}$ & $\mathrm{p}$ \\
\hline Audición & .52 & 1.39 & -2.81 & 19 & .011 \\
& $(.16)$ & $(1.35)$ & & & \\
Verbal & .52 & 1.17 & -3.32 & 19 & .004 \\
& $(.12)$ & $(.95)$ & & & \\
Lenguaje & .52 & 1.28 & -3.58 & 19 & .002 \\
& $(.13)$ & $(.99)$ & & & \\
\hline
\end{tabular}


Tabla $\mathbf{N}^{\circ} 9$

VABS medias valores desarrollo pre and postest, desviación estándar y puntajes t-test

\begin{tabular}{lccccc}
\hline Subpuntajes & Valor 1 & Valor 2 & $\mathrm{t}$ & $\mathrm{df}$ & $\mathrm{p}$ \\
\hline Comunicación & .41 & .90 & -2.95 & 19 & .008 \\
Vida Diaria & $(.16)$ & $(.81)$ & & & \\
& .63 & .56 & .490 & 19 & $\mathrm{~ns}$ \\
Socialización & $(9.94 \mathrm{E}-02)$ & $(.61)$ & & & \\
& .50 & .74 & -1.13 & 19 & $\mathrm{~ns}$ \\
Motor & $(.16)$ & $(.89)$ & & & \\
& .87 & .37 & 2.98 & 19 & .008 \\
& $(.14)$ & $(.72)$ & & & \\
\hline
\end{tabular}

\section{REFERENCIAS}

Alpern, G.A., Boll, T., \& Shearer, M. (1989). Developmental Profile II. Los Angeles, CA: Western Psychological Services.

Anderson, S.R., Avery, D.L., DiPietro, E.K., Edwards, G.L., \& Christian, W.P. (1987). Intensive home-based early intervention with autistic children. Education and Treatment of Children, 10 (4), 352-366.

COSAC. (1994). I know this child can learn! The XYZ's of behavioral intervention: Reaching and teaching individuals with autism. Ewing, NJ.

Dyer, K. (1989). The effects of preference on spontaneous verbal requests in individuals with autism. Journal of the Association of Persons with Severe Handicaps, 14 (3), 184-189.

Glover, M.E., Preminger, J.L., \& Sanford, A.R. (1988). The early learning accom- plishment profile for young children. Chapel Hill, NC: Chapel Hill TrainingOutreach Project.

Green, G. (1996). Early behavioral intervention for autism: What does research tell us? En: C. Maurice, G. Green, \& S.C. Luce (Eds.), Behavioral intervention for young children with autism: A manual for parents and professionals (pp. 29-44). Austin, TX: Pro-Ed.

Harris, S.L., Handleman, J.S., Gordon, R., Kristoff, B., \& Fuentes, F. (1991). Changes in cognitive and language functioning of preschool children with autism. Journal of Autism and Developmental Disorders, 21, 281-290.

Harris, S.L., Handleman, J.S., Kristoff, B., Bass, L., \& Gordon, R. (1990). Changes in language development among autistic and peer children in segregated and integrated preschool settings. Journal of Autism and Developmental Disorders, 20, 23-21. 
Helm, D.T., \& Kozloff, M.A (1986). Research on parent training: Shortcomings and remedies. Journal of Autism and Developmental Disorders, 16 (1), $1-22$.

Holmes, D.L. (1990-1991). (Exec. Ed.) Eden Institute Curriculum Series. Volumes 1-5.

Huff, R.C. (1996). Community-based early intervention for children with autism. En: C. Maurice, G. Green, \& S.C. Luce (Eds.), Behavioral intervention for young children with autism: A manual for parents and professionals (pp. 251265). Austin, TX: Pro-Ed.

Jacobson, J. W., Mulick, J.A., \& Green, G. (November, 1996). Risk-cost-benefit analysis of intensive early intervention for young children with autism. (Via me-list@listserv.iupui.edu.)

Koegel, R.L., Dyer, K., \& Bell, L. (1987). The influence of child-preferred activities on autistic children's social behavior. Journal of Applied Behavior Analysis, 20, 243-252.

Koegel, R.L., Koegel, L.K., \& Surratt, A. (1992). Language intervention and disruptive behavior in preschool children with autism. Journal of Autism and Developmental Disorders, 22 (2), 141153.

Krug, D.A., Arick, J.R., \& Almond, P.J. (1980). ASIEP: Autism Screening Instrument for Education Planning. Austin, TX: Pro-Ed.

Lovaas, O.I. (1981). Teaching developmentally disabled children: The me book. Austin, TX: Pro-ed.

Lovaas, O.I. (1987). Behavioral treatment and normal education and intellectual functioning in young autistic children.
Journal of Clinical and Consulting Psychology, 55, 3-9.

McEachin, J.J., Smith, T., \& Lovaas, O.I. (1993). Long-term outcome for children with autism who received early intensive behavioral treatment. American Journal on Mental Retardation, 97 (4), 359-372.

Rimland, B. (1995). Is there an autism epidemic? Autism Research Review International, 9 (3), 3.

Schopler, E., \& Reichler, R.J., \& Renner, B.R. (1990). The childhood autism rating scale (CARS). Los Angeles, CA: Western Psychological Services.

Sparrow, S.S., Balla, D.A., \& Cicchetti, D.V. (1984). Vineland Adaptive Behavior Scales (Interview edition). Circle Pines, MN: American Guidance Service, Inc.

Zimmerman, I.L., Steiner, V.G., \& Pond, R.E. (1979). Preschool Language Scale. The Psychological Corporation: Harcourt Brace Jovanovich, Inc. 